\title{
Philosophiques
}

\section{Critique de la théorie marxiste de l'État}

\section{Robert Tremblay}

Volume 13, numéro 2, automne 1986

URI : https://id.erudit.org/iderudit/203320ar

DOI : https://doi.org/10.7202/203320ar

Aller au sommaire du numéro

Éditeur(s)

Société de philosophie du Québec

ISSN

0316-2923 (imprimé)

1492-1391 (numérique)

Découvrir la revue

Citer cet article

Tremblay, R. (1986). Critique de la théorie marxiste de l'État. Philosophiques, 13(2), 267-289. https://doi.org/10.7202/203320ar

\section{Résumé de l'article}

La théorie marxiste de l'État souffre de l'ambiguïté fondamentale de la doctrine marxiste. C'est sur la question centrale de la " période de transition » et du " dépérissement » de l'État prolétarien que cette théorie révèle les apories d'une conception économiste des superstructures. Par une étude de certains textes classiques sur la question, nous tentons de démontrer que la dérive totalitaire des états socialistes procède tant de l'incapacité de poser le problème de la bureaucratisation de l'État que de la croyance mythique en une extinction spontanée de l'État. Cette théorie reste aveugle aux conséquences d'une concentration étatique des pouvoirs qu'elle préconise néanmoins explicitement. Ainsi le marxisme en vient-il à combler la figure hégélienne d'un État représentant l'universalité. 


\title{
CRITIQUE DE LA THÉORIE MARXISTE DE L'ÉTAT
}

\author{
par Robert Tremblay
}

\begin{abstract}
RESUME. La théorie marxiste de l'État souffre de l'ambiguïté fondamentale de la doctrine marxiste. C'est sur la question centrale de la «période de transition» et du «dépérissement» de l'État prolétarien que cette théorie révèle les apories d'une conception économiste des superstructures. Par une étude de certains textes classiques sur la question, nous tentons de démontrer que la dérive totalitaire des états socialistes procède tant de l'incapacité de poser le problème de la bureaucratisation de l'État que de la croyance mythique en une extinction spontanée de l'État. Cette théorie reste aveugle aux conséquences d'une concentration étatique des pouvoirs qu'elle préconise néanmoins explicitement. Ainsi le marxisme en vient-il à combler la figure hégélienne d'un État représentant l'universalité.
\end{abstract}

ABSTRACT. The Marxist theory of the State is afflicted by the fundamental ambiguity of the Marxist doctrine. It is in relation with the central question of the "transition period" and of the "witering away » of the proletarian State that this theory discloses the difficulties of an economic determinism of the superstructure. Through a study of some classical texts on this subject, we will try to demonstrate that the totalitarian drift of the Socialist States proceeds from the incapacity to set forth the problem of the bureaucratisation of the State as well as from a mythical belief in a spontaneous extinction of the State. This theory stays blind to the consequences of a concentration of powers in the State, although it advocates it explicitly. Marxism thus fulfils the Hegelian figure of a State representing universality.

«L'essence de l'État, c'est l'universel en et pour soi, l'élément rationnel de la volonté, subjectif toutefois en tant qu'il se sait et s'affirme, et un individu en sa réalité. D'une manière générale, son œuvre par rapport à l'extrême de l'individualité, c'est-à-dire la foule des individus, est double; il doit d'abord les conserver comme personnes, faire par suite du droit une réalité nécessaire, puis avancer leur bienêtre auquel chacun travaille pour lui-même, mais qui a un côté 
général; il doit protéger la famille et diriger la société civile - en second lieu il doit ramener ces deux choses ainsi que toute la mentalité et toute l'activité de l'individu, qui tend à se constituer son propre centre, à la vie de la substance universelle et en ce sens faire obstacle comme puissance libre à ces spbères qui lui sont subordonnées et les conserver dans leur immanence substantielle.»

Hegel, Précis de l'Encyclopédie des

Sciences Philosophiques, \# 257

\section{LES SUPERSTRUCTURES DANS LA THÉORIE MARXISTE}

Malgré un renversement tardif de l'interprétation économiste du marxisme par Engels ${ }^{1}$, et malgré quelques déclarations de Marx lui-même ${ }^{2}$, il y a assurément un noyau d'économisme indéracinable dans le marxisme, lequel ne saurait être plus explicite que dans la formule de la «détermination en dernière instance». D'où la question des superstructures, qui prendra une grande importance, de Gramsci à Althusser ${ }^{3}$, dans le processus de renouvellement de la pensée marxiste au vingtième siècle; mais il s'agit là de penseurs dont l'importance est toute relative face au réductionnisme brutal d'un Staline. Si l'économisme apparaît à plusieurs, marxistes ou libéraux, comme une théorie crédible des rapports sociaux, c'est que nous vivons sous l'effet de la fétich isation marchande des rapports sociaux.

Il y a chez Marx une ambivalence fondamentale entre une conception volontariste et une conception déterministe de l'histoire. S'il écrit, à propos du prolétariat : «Son action commune, (...), est une des premières conditions de son émancipation.» ${ }^{4}$; renchérissant ainsi sur sa conception de la lutte des classes comme «moteur de l'histoire»; s'il rappelle dans Le Capital, que les catégories économiques elles-mêmes ne sont que l'expression de rapports sociaux déterminés; on retrouvera cependant sous sa

1. F. EnGels, (2), p. 11, 21-22 septembre 1890, lettre à Bloch. (Les chiffres entre parenthèses renvoient à la liste des ouvrages cités.)

2. Qu'on se rappelle simplement la troisième thèse sur Feuerbach : «La coïncidence du changement des circonstances et de l'activité humaine ne peut être considérée et comprise rationnellement qu'en tant que pratique révolutionnaire».

3. On pense ici en particulier au double concept de l'État chez Gramsci, l'État politique et l'État comme formation sociale-historique, au concept d'appareil idéologique et enfin à la théorie althussérienne de l'idéologie.

4. K. MARX, (6), p. 56. 
plume des formules du plus pur positivisme. Par exemple il écrit: «... le développement de la formation économique de la société est assimilable à la marche de la nature et à son histoire...» ${ }^{5}$, où l'on constate un déterminisme économique extrêmement rigide. Ce déterminisme apparaît surtout dans les œuvres de maturité à propos de l'économie politique; par contre Marx fait sa part à l'autonomie du politique, à la lutte et à la conscience de classe, dans ses pamphlets et ses études historiques.

Il y a une oscillation patente dans l'œuvre des premiers marxistes entre un positivisme naturaliste, où le mouvement de l'histoire est essentiellement dépendant du développement des forces productives et où sa marche doit suivre des étapes précises liées à la transformation des modes de production; et un volontarisme révolutionnaire, où la volonté et la conscience de classe jouent le rôle fondamental. Les marxistes «conséquents» ont tenté de réconcilier les deux points de vue par un usage particulier de la dialectique : on distinguera par exemple le pôle déterminant de la contradiction, (l'instance économique est déterminante en dernière instance), et son pôle dominant (la lutte et la conscience de classes peuvent devenir un facteur dominant) ${ }^{6}$. Mais cette "entourloupette» théorique masque mieux le problème qu'elle ne le résoud: l'économisme en ressort légitimé alors que l'autonomie des superstructures demeure impensée.

Marx avait aperçu la diff iculté, aussi présente-t-il l'articulation de la lutte des classes au développement de la formation économique de la société comme un rapport d'accélération ou de décélération de l'histoire: l'histoire a un sens (capitalismesocialisme-communisme) et l'action des hommes sur l'histoire consiste à stimuler ou à inhiber l'action interdépendante mais autonome des déterminations économiques. Cette solution reconduit à un autre niveau l'ambivalence; en effet pourquoi devrait-on

5. K. MARX, (7), «Préface à la première édition allemande», p. 13.

6. Selon cette conception toute contradiction comprendrait deux oppositions : celle qui sépare les pôles dominant et dominé de la contradiction (par exemple les classes sociales), et celle qui sépare les pôles déterminant et déterminé de la contradiction (la base économique est déterminante alors que les superstructures sont déterminées). Un facteur non déterminant, par exemple la «conscience de classe», facteur idéologique, peut devenir le pôle dominant de la contradiction si les conditions économiques générales sont mûres pour une révolution, c'est-à-dire qu'il peut être le facteur décisif du développement de la contradiction. 
lutter en vue d'un dénouement qui est de toute manière inévitable? C'est ce raisonnement qui soutiendra à l'origine la déviation sociale-démocrate (la Deuxième Internationale). D'où la surenchère léniniste dont le caractère est essentiellement moral: il faut faire la révolution pour abréger la souffrance des masses, mettre fin à l'exploitation et, - tautologie proprement idéologique - , suivre la voie du marxisme. Bref il faut lutter pour accomplir la prophétie. Mais cette ligne de combat ne peut ni lever l'ambivalence, ni compenser pour l'absence d'une théorie de l'autonomie $\mathrm{du}$ politique.

S'il y a un intérêt à questionner l'ensemble de la problématique des superstructures, la situation politique actuelle et la conscience grandissante du caractère bureaucratique des états socialistes ${ }^{7}$, nous incite à tourner tout d'abord notre regard sur la théorie marxiste de l'État telle qu'elle apparaît chez ses fondateurs, (Marx, Engels et Lénine), plutôt que vers l'œuvre novatrice, mais somme toute périphérique, des révisionnistes (Lukacs, Gramsci, Korsch et Castoriadis, pour n'en nommer que quelques-uns), afin de chercher à dépister si un défaut théorique ne présiderait pas à la dérive totalitaire des États inspirés de la doctrine marxiste. Sans chercher à résoudre ainsi la question, qui comporte évidemment bien d'autres aspects - n'en déplaise aux «nouveaux philosophes»-, nous chercherons brièvement à cerner le paradoxe de la théorie marxiste de l'État.

\section{LA THÉORIE DE L'ÉTAT DE KARL MARX}

Contrairement à ce qu'en pense Lefebvre ${ }^{8}$, Marx et Engels ont élaboré une théorie de l'État parfaitement cohérente avec l'ensemble de leur doctrine. Qu'elle n'ait jamais été exposée systématiquement, voilà un fait évident, mais n'en est-il pas de même de la dialectique dont Lefebvre est le premier à affirmer l'importance et la prépondérance dans le corpus marxien? D'ailleurs, comme dans le cas du concept de dialectique, cette absence de systématisation est révélatrice d'un trouble dans la

7. On pourra se référer aux travaux de Castoriadis, Lefort et du groupe Socialisme ou Barbarie.

8. H. LEFEBVRE, (3), en particulier le chapitre VII. 
doctrine. Par ailleurs Lefebvre a raison d'indiquer que toute la conception marxiste s'effondre si on l'ampute de l'idée du communisme comme fin de l'État et donc fin du politique.

De manière générale on peut dire que pour Marx le communisme ne sera jamais pensé comme réalisation immédiate; au contraire ce sera justement l'une des distinctions fondamentales avec le socialisme dit utopique, l'avènement du communisme nécessitera une période de transition pendant laquelle un État de transition, un État ouvrier et socialiste accomplissant la dictature du prolétariat, préparera son propre dépérissement. Il faut donc distinguer trois périodes:

1) la destruction de l'État bourgeois (ou période révolutionnaire proprement dite);

2) le socialisme et la dictature du prolétariat (ou période de transition);

3) le communisme, caractérisé par la «libre association de producteurs» et l'absence de la division de la société en classes antagoniques.

Ce qui fait problème à notre sens, théoriquement et pratiquement, c'est la question du passage de la deuxième à la troisième période, soit la question du dépérissement et de l'extinction de l'État.

\subsection{Le Manifeste}

Dans le Manifeste du Parti Communiste (1847-48), on peut lire que le prolétariat, lorsqu'il aura acquis la suprématie politique, s'en prévaudra pour arracher tout le capital à la bourgeoisie et « ... pour centraliser tous les instruments de production entre les mains de l'État, c'est-à-dire du prolétariat organisé en classe dominante...» ${ }^{9}$ On voit ici que la conception originaire de Marx, loin d'exclure le socialisme d'État le prescrit explicitement. D'où on ne peut suivre Lefebvre lorsqu'il attribue cette interprétation du socialisme exclusivement à Lasalle, Engels et Staline, dans une intention trop évidente de sauver son maître à penser. Surenchérissant sur ce point de vue, Marx critiquera la position anarchiste de la disparition simultanée de l'État bourgeois et de toutes les

9. K. MARX, (6), p. 58. Nous soulignons. 
autres formes d'organisation étatique, préconisant plutôt " pour les pays les plus avancés» une série de mesures de renforcement de l'État : centralisation et concentration du pouvoir aux mains de l'État, propriété étatique du sol, expropriation de la rente foncière, monopole étatique sur le crédit et la banque nationale, contrôle du transport et enfin planification économique et industrielle centralisée. La seule exception concernera les forces de répression (armée et police), puisqu'il suppose que la révolution aura transformé la classe ouvrière toute entière en armée révolutionnaire exerçant directement le contrôle social. Comme cette «armée» agit sous la direction du Parti Communiste, on peut voir poindre une structure étatique bicéphale où le pouvoir ultime est aux mains du Parti.

Un esprit éclairé pourrait voir là un danger pour la démocratie populaire dont cet État tient sa légitimité. Marx répondra simplement que «... le pouvoir public perd son caractère politique ${ }^{10} . »$ On touche là un point fondamental: comment un pouvoir public peut-il ne pas être politique? La réponse relèvera de ce que Marx appelle ailleurs une mauvaise abstraction. Puisque le pouvoir politique se définit comme le pouvoir d'une classe dominante dont l'État est l'instrument de domination, et que le prolétariat en abolissant le régime capitaliste de production abolit «... les conditions de l'antagonisme de classe, il détruit les classes en général et, par là même, sa propre domination comme classe ${ }^{11}$.» Pourtant les conditions de cette suppression de l'État par l'autodestruction du prolétariat, de même que la destruction corrélative de l'antagonisme de classe, ne sont définies nulle part: le postulat selon lequel le prolétariat sera la dernière classe de l'histoire reste infondé. Mais il a une origine, dans les Manuscrits de 1844, sur laquelle il serait trop long de s'arrêter ici; retenons seulement que le prolétariat y apparaît comme la première classe universelle de l'histoire, celle qui émancipera l'humanité parce qu'elle n'a rien à perdre que ses chaînes, incarnation vraie, - parce qu'absolument dépouillée - , de l'humanité générique : c'est en un sens profondément hégélien la classe universelle en soi et pour soi destinée par son propre mouvement à devenir

10. K. Marx, F. Engels, (6), p. 60.

11. Ibid., p. 60. 
effectivement réelle, i.e. à réaliser son universalité. À cet égard l'État apparaîtra comme la médiation particulière qui incarne provisoirement l'universel en devenir; mais puisqu'il s'agit d'un universel particularisé, il ne saurait se maintenir autrement que comme la promesse d'un universel réalisé : voilà le secret, c'est par fidélité à la métaphysique dialectique de Hegel (mais renversée) que Marx postule le dépérissement de la classe prolétarienne et de son incarnation étatique.

De plus on sait que pour Marx les communistes «... n'ont point d'intérêts qui les séparent du prolétariat» ${ }^{12}$, donc les communistes seront les dépositaires de l'universalité non réalisée du prolétariat, d'abord au sein du Parti, puis dans l'État. On voit donc clairement dans le Manifeste une conception du prolétariat et de l'État telle que toutes les conditions théoriques de la domination d'une minorité «communiste» sur la société dans son ensemble par le biais du pouvoir d'État soient présupposées.

\subsection{La Guerre civile en France (1871)}

C'est dans la Commune de Paris que Marx verra la concrétisation de ses thèses, mais celles-ci s'infléchiront sensiblement sous l'épreuve des faits. En effet la Commune ${ }^{13}$ lui semble être une forme politique nouvelle qui contredit totalement la forme étatique; ainsi la période post-révolutionnaire ne devra plus être conçue comme devant mener à la constitution d'un nouvel État. Ici Marx semble se rapprocher des conceptions anarchistes qui ont toujours nié la nécessité d'une transition étatiste. Pour Marx la Commune est une «forme politique tout à fait susceptible d'expansion, tandis que toutes les formes antérieures de gouvernement avaient été essentiellement répressives. Son véritable secret le voici : c'est essentiellement un gouvernement de la classe ouvrière...» ${ }^{14}$ Organe agissant, à la fois législatif et exécutif, la Commune récupère toute l'initiative qui appartenait

12. Ibid., p. 49.

13. On considérera ici comme équivalents les termes de commune, conseil et soviet, référant par là de manière générale au socialisme conseilliste (i.e. à une forme de démocratie directe).

14. K. MARX, (8), p. 67. 
autrefois à l'État. La transition disparaît pratiquement, la Commune est le paradigme du communisme immédiatement réalisable. Par exemple, soulevant la question du contrôle de la production nationale, Marx affirme que la Commune voulait briser le monopole de la "classe de expropriateurs» et confier la tâche d'établir un "plan commun» à l'ensemble des "associations coopératives»; tout à son enthousiasme il s'exclame: «que serait-ce, messieurs, sinon du communisme, du très "possible" communisme? " $15 \mathrm{La}$ "Constitution Communale» serait donc la forme spécifique d'exercice du pouvoir prolétarien et cette forme ne pourrait pas être vue comme une forme étatique, au contraire celle-ci devrait restituer au corps social «... toutes les forces jusqu'alors absorbées par l'Etat parasite qui se nourrit sur la société et en paralyse le mouvement ${ }^{16}$. . Ainsi la révolution du peuple en armes en réalisant la Commune abolit l'Etat, aussi une théorie de la transition est-elle inutile puisque dans la logique marxiste il ne saurait y avoir désormais d'État de transition. Nous voyons ici en partie, - mais en partie seulement - , pourquoi le problème de la transition restera impensé, bien que cette position de Marx ne soit guère caractéristique d'une attitude plus générale qu'il adoptera devant ce problème, comme Le Manifeste, la Critique du Programme de Gotha et Le Capital le montrent suffisamment.

Engels dans une introduction à l'ouvrage de Marx se fera plus hésitant et en quelque sorte plus «marxiste». Si la position de Marx semble pencher vers l'anarchisme, il y a fort à parier que c'est essentiellement pour des raisons conjoncturelles, un certain aveuglement révolutionnaire n'étant pas étranger, par ailleurs, à son analyse de la Commune. Selon Engels la Commune, «ce pouvoir nouveau, vraiment démocratique», ne rend pas l'État tout à fait inutile, car il faut exercer une répression contre l'ancienne classe dominante jusqu'à sa disparition. Si l'Etat est rendu caduc, historiquement parlant, c'est un «mal nécessaire» dont le prolétariat devra néanmoins s'accomoder s'il veut détruire les derniers vestiges de la domination antérieure. La dictature du prolétariat sera donc étatique.

15. Ibid., p. 68.

16. Ibid., p. 66. Nous soulignons. 
On ne saurait trop insister sur les difficultés pratiques de cette position; en effet rien ne garantit que l'État ainsi constitué saura reconnaître ses véritables ennemis de classe, ni même qu'il exercera sa répression exclusivement contre ceux-ci, et par-dessus tout rien — ni dans l'histoire, même antérieure à ce texte, ni dans la logique du pouvoir étatique, telle qu'analysée par les marxistes eux-mêmes - ne devrait nous porter à croire qu'il consentira à disparaître. De fait si Marx dans son analyse de la Commune de Paris infléchit sa position vers un communisme des conseils, vers l'idée d'une démocratie directe, celle des producteurs associés, Engels nous rappelle que face au problème de la répression (et, comme on le verra plus loin, ceux de l'organisation) la position anarchiste ne saurait suffire, car la répression est affaire de professionnels. C'est pour cette première raison que le socialisme d'État l'emportera dans la théorie comme dans la pratique marxistes.

\subsection{La Critique du Programme de Gotha (1875)}

Dans la Critique, si l'État est bel et bien cet organisme détestable qu'il faut détruire, car sa seule fonction consiste à réprimer le peuple et à organiser autoritairement la vie sociale en se finançant à même le surtravail, l'État subsistera néanmoins pour un certain temps car: "Entre la société capitaliste et la société communiste, se place la période de transformation révolutionnaire de celle-là à celle-ci. $\grave{A}$ quoi correspond une période de transition politique où l'État ne saurait être autre chose que la dictature révolutionnaire du prolétariat ${ }^{17}$.» À propos de cette transition Marx écrira que le rapport Etat-société sera renversé, l'État sera subordonné à la société ${ }^{18}$. Mais il se fait prudent

17. K. MARX, (9), p. 26. Nous soulignons.

18. Remarquons au passage comment Marx demeure à l'intérieur de l'opposition hégélienne de la société civile et de l'État, inversant simplement les termes de l'apologétique de Hegel où l'État apparaissait comme garant de la sécurité et de la liberté.

Pour être plus précis il faut ajouter le troisième terme de la trilogie hégélienne : État, société civile et famille. L'attitude de Marx envers la famille, comme envers la sphère de la "vie privée», est peu explicite; certains passages nous porteraient à croire que selon lui le socialisme devrait libérer la famille des contraintes étatiques et rendre la vie privée aux individus. Mais on peut supposer qu'un raisonnement semblable à celui que nous tenons sur l'Etat nous mènerait à des conclusions similaires: en définitive le socialisme marxiste accomplit l'idéal hégélien. 
refusant de préciser quelles fonctions sociales assumées par l'État seraient maintenues dans une société communiste. Marx manque de précision ici; il nous faudrait savoir en quel sens, sous quels rapports et comment l'État serait-il soumis à la société. De la même façon la question de savoir quelles fonctions sociales étatiques la société communiste devra maintenir intéresse-t-elle au plus haut point la question du communisme lui-même. Bien sûr les marxistes répondront qu'ils ne sont ni prophètes ni utopistes, mais cette réponse ne peut nous satisfaire en ce sens où leur appel à l'action ne saurait être purement l'expression d'une nécessité historique et que dans cette exacte mesure les «masses» sont en droit de connaître la direction que leur « avant-garde» privilégiera. A moins que l'on ne pense qu'il s'agisse en effet d'une nécessité historique, mais alors l'action politique n'a plus guère de sens, ni la théorie qui la guide.

Dans une lettre à Bebel sur la Critique, Engels précise encore: l'État demeurera mais il ne sera qu'une «institution temporaire» destinée à réprimer les adversaires du prolétariat. «Il conviendrait d'abandonner tout ce bavardage sur l'Etat...», écrit-il, car « ... le jour où il devient possible de parler de liberté, l'État cesse d'exister comme tel ${ }^{19}$." Que voilà donc un beau raisonnement! S'il y a l'État, il n'y a pas de liberté; or il y a liberté; donc il n'y a pas l'État! Examinons la prémisse : là où il y a l'État il n'y a pas de liberté; nous conviendrons aisément que l'État comporte la répression et que la répression signifie un moins de liberté, mais justement comment l'État prolétarien pourrait-il être un État, donc comporter une répression, - ce que les marxistes admettent - , et en même temps n'être pas un État «comme tel», puisqu'il suppose la liberté ? Le plus simplement du monde: en étant répressif, comme tous les États, mais en dégageant un espace nouveau de liberté, justement pour la classe dominée ${ }^{20}$. Comme cette liberté ne saurait être absolue, - puisqu'il y a un Etat, même minimal -, alors l'État continue d'exister. La question reste entière.

19. F. ENGELS, (9), p. 41, 18-28 mars 1875.

20. Cet espace de liberté est évidemment tout relatif, la nature répressive et oppressive de l'État dans l'exécution de ses fonctions de contrôle social étant une caractéristique universelle. Notre formulation n'a ici aucune portée empirique, elle est acceptée uniquement pour les fins de la discussion. 
Commune ou communauté (Gemeinweisen), la forme de transition est mal dégagée par Engels. Chez Marx l'ambiguité persiste: entre la «commune» et «l'État», la forme politique de la dictature du prolétariat reste obscure. Plus profondément Marx s'interdit de questionner la transition qu'il considère pourtant inévitable. Or le problème qui se pose est pourtant clair: comment l'État, force oppressive par définition, acceptera-t-il de se liquider? C'est par un recours à son économisme structurel que Marx arrive à oblitérer la question: l'État ne fait que refléter la société qui est déterminée en dernière instance par son infrastructure économique (le mode de production, la division du travail, le développement des forces productives, etc.). On ne peut traiter l'État «... comme une réalité indépendante, possédant ses propres "fondements intellectuels, moraux et libres"» ${ }^{21}$. Ainsi lorsque le mode de production socialiste se développera, il entraînera un dépérissement automatique de l'État.

Nous nous retrouvons toujours devant une pétition de principe: le socialisme suppose le dépérissement de l'État, donc l'État dépérira! Il y a plus encore chez Marx une impossibilité de penser l'autonomie de l'État. Sans théorie de la spécificité de l'État, sans théorie de la transition, le marxisme sera peu préparé à affronter le problème politique majeur qui sera le sien: la transformation de la «dictature du prolétariat» en dictature bureaucratique d'État.

\section{Engels : le travail d'un épigone}

Il importe au plus haut point de clarifier la relation qui est établie entre l'infrastructure économique et la superstructure politique, dans la mesure où c'est du caractère inégal et asymétrique de celle-ci que le marxisme infère la dépendance de l'État et de son dépérissement éventuel envers la transformation des « conditions matérielles d'existence». Plus précisément on peut se demander de quelle manière l'État reflète-t-il la structụre économique de la société. C'est autour de ces questions qu'Engels est le plus éclairant.

21. K. MARX, (9), p. 25. 


\subsection{Lettres sur le matérialisme historique}

C'est assez tardivement que Marx et Engels comprendront le danger d'une mésinterprétation de leurs thèses sur la détermination économique de dernière instance ${ }^{22}$. Ainsi Engels écrira-t-il :

C'est Marx et moi-même, partiellement, qui devons porter la responsabilité du fait que, parfois, les jeunes donnent plus de poids qu'il ne lui est dû au côté économique. Face à nos adversaires, il nous fallait souligner le principe essentiel nié par eux, et alors nous ne trouvions pas toujours le temps, le lieu, ni l'occasion de donner leur place aux autres facteurs qui participent de l'action réciproque ${ }^{23}$.

Faire de l'économique le seul facteur déterminant est proprement "absurde», écrit-il; les divers éléments de la superstructure agissent sur l'issue des luttes historiques et même en déterminent la forme à travers une série de facteurs plus ou moins contingents (par exemple des particularités nationales ou étatiques). Le mouvement économique cependant finit toujours par se frayer un chemin comme une nécessité incontournable. L'action en retour des facteurs superstructurels sur la base économique, Althusser la nomme surdétermination, terme qui manifeste particulièrement bien la secondarité de cette action en retour par rapport à l'action déterminante de l'infrastructure économique ${ }^{24}$.

L'État naît de la division du travail et de la scission de la société en classes antagoniques; la société de classes définit pour l'État un certain nombre de fonctions permanentes dont elle ne peut se dispenser, ainsi les mandataires du pouvoir d'État acquièrent-ils des intérêts particuliers et développent une « nouvelle force indépendante» ${ }^{25}$. La société dans son ensemble doit subir le contre-coup du mouvement relativement indépendant du pouvoir d'État; il en va de même du droit. «La violence (c'est-à-dire le pouvoir d'État) est, elle aussi, une puissance économique !» ${ }^{26} \mathrm{On}$ voit clairement par cette métaphore à la fois la volonté d'Engels de penser l'action autonome de l'État dans la dynamique historique,

22. La formulation la plus célèbre de ce déterminisme se retrouve dans la Préface de la Contribution à la critique de l'économie politique, de 1859.

23. F. ENGELS, (2), p. 11, 21-22 sept. 1890.

24. L. Althusser, Pour Marx, F. Maspéro, 1965.

25. F. ENGELS, (2), p. 16,27 oct. 1890.

26. Ibid., p. 22. 
et son impossibilité de la penser autrement qu'à travers le postulat de la détermination économique. Les forces économiques donnent au mouvement historique son contenu le plus réel, bien que celui-ci soit la plupart du temps «inconscient», alors que l'efficacité propre de l'État est rapportée aux seules formes; donc à l'inessentiel, à la contingence, à ce qui apparâ̂t en surface du mouvement historique. Par exemple lorsque Engels examine l'effet proprement économique des politiques étatiques, il enferme l'action de l'État dans la dichotomie suivante: ou bien elle va dans le sens des forces économiques et accélèrent leur mouvement, ou bien elle s'y oppose et se condamne à la faillite! On voit que Engels demeure incapable de concevoir une action étatique véritablement indépendante, et favorise ainsi l'économisme qu'il combat par ailleurs; en effet il ne suffit pas d'affirmer l'existence d'autres facteurs, encore faut-il en montrer l'action spécifique dans chaque cas (à cet égard on pourrait tenir un raisonnement similaire à propos de la culture) et leur relative autonomie systémique, plutôt que de les rapporter à l'économique comme on ramène des existants à leur essence.

\subsection{L'Anti-Dühring}

Si le pouvoir d'État fonctionne à la violence, c'est qu'il doit assurer le maintien des «conditions extérieures générales du mode de production" ${ }^{27}$, dont prioritairement la soumission des classes exploitées. Si, en quelque sorte, l'État représente l'ensemble de la société et les intérêts communs à toutes les classes, c'est dans la mesure où, d'une part, il est un outil aux mains d'une classe dominante qui voit à présenter ses intérêts particuliers comme universels, et, d'autre part, dans la mesure où ces intérêts particuliers comportent nécessairement un élément de généralité.

L'État moderne, selon Engels, pousse le rapport capitaliste à son comble et, arrivé à ce point, «il se renverse ${ }^{28}$. En effet la propriété d'État « révélant» le caractère collectif de la production et le caractère social des moyens de production, renferme en lui ce qu'il appelle le moyen formel de dépasser le capitalisme.

27. F. ENGELS, (1), p. 315.

28. Ibid. 
Lorsque le prolétariat s'emparera du pouvoir d'État, il transformera la propriété privée des moyens de production en propriété d'État (sic!). Ce nouvel élément est fort révélateur: non seulement «l'État de transition» aura-t-il un appareil répressif et une administration publique, mais en plus il sera propriétaire de tous les moyens de production. Quelle concentration formidable de richesse et de puissance! On a peine à comprendre comment après un tel aveu les marxistes ont pu croire encore au «dépérissement de l'État». Mais il y a plus grave: Engels confirme dans le même texte la possibilité d'existence d'un capitalisme collectif d'État, voire même d'un capitalisme sans capitalistes (il donne comme exemple les sociétés par actions); l'équation est simple: un Etat propriétaire exclusif de tous les moyens de production devient un capitalisme "collectif», voire un capitalisme sans capitalistes, il en est même la forme la plus viable puisqu'il concentre aussi les pouvoirs répressifs et administratifs. Cela les marxistes l'entrevoyaient peut-être, mais pour eux il y avait plus important : la lutte des classes qui elle-même origine du procès de travail. Ainsi la transformation du mode de production assurait-elle pour eux la disparition des conditions économiques de l'antagonisme de classes; or sans classe oppressive l'État devait disparaître ; c'était sans compter l'apparition d'une classe bureaucratique d'État.

Le dogme d'une classe prolétarienne s'abolissant elle-même (car la classe absolument dominée sera la classe universelle) permet d'éviter une question qui découle directement des prémisses que Engels avait énoncées : l'État prolétarien ne risque-t-il pas de dégénérer en un nouvel État capitaliste «collectif» à la solde de ses dirigeants?

\section{Engels écrit :}

Le premier acte par lequel l'État apparaît comme le représentant de toute la société, - la prise de possession des moyens de production au nom de la société -, est en même temps son dernier acte propre en tant qu'État.

L'intervention d'un pouvoir d'Etat dans des rapports sociaux devient superflue dans un domaine après l'autre, et entre alors naturellement en sommeil. 
Le gouvernement des personnes fait place à l'administration des choses et à la direction des opérations de production. L'Etat n'est pas «aboli», il s'éteint. ${ }^{29}$

Engels présente cette théorie de l'extinction de l'État comme une alternative au socialisme d'État et à la théorie anarchiste de l'abolition de l'État. Pourtant elle fait problème à plusieurs égards. Premièrement il est extrêmement paradoxal, du point de vue marxiste, d'opposer le «gouvernement des personnes» à «l'organisation des opérations de production», puisque l'un des traits spécifiques de l'aliénation du travail est justement l'absence de contrôle direct des travailleurs sur l'organisation du travail, or celle-ci devient une prérogative de l'État, non des travailleurs eux-mêmes. Ainsi l'État continuerait-il par ce biais à " gouverner des personnes». Deuxièmement on remarquera que le mode de propriété caractéristique du socialisme devient la propriété étatique; lorsque l'on connaît l'insistance de Marx sur le fait que les formes de propriété reflètent l'état social dans son ensemble (à chaque mode de production correspond une forme spécifique de propriété), il nous faut reconnaître que la propriété étatique représente un certain mode de production, qui n'est pas le communisme, et donc est susceptible d'une certaine permanence. Troisièmement ce qui caractérise en partie la bourgeoisie c'est qu'elle s'assure la direction des opérations de production au détriment des producteurs eux-mêmes ; le fantôme du capitalisme d'État nous hanterait alors à nouveau justement à cause du transfert d'autorité vers l'État. Quatrièmement Engels n'explique pas comment l'État se retirerait graduellement de tous ses champs d'intervention, ni pourquoi, sinon que cette intervention deviendrait «superflue»: mais alors de quel point de vue le devient-elle?

Enfin, c'est le nœud de la question, nous devons nous demander qu'est-ce qui garantit l'extinction de l'État prolétarien. Engels répond que c'est le développement de la production et plus généralement le passage du règne de la nécessité au règne de la liberté. "Les lois de leur propre pratique sociale, écrit-il, qui, jusqu'ici, se dressaient devant eux comme des lois naturelles, étrangères et dominatrices, sont dès lors appliqués par les 
hommes en pleine connaissance de cause, et par là dominées ${ }^{30}$.» Engels renvoie à l'organisation planifiée de la production pour illustrer cette transformation. $\AA$ nouveau l'argument marxiste repose sur un raisonnement circulaire: l'État, instrument de domination de classe, s'éteint parce qu'on accède à la société sans classes ; on accède à la société sans classe au moment où, devenu inutile, l'État s'éteint. La clef de ce raisonnement se trouve ici : «La scission de la société en une classe exploiteuse et une classe exploitée... était une conséquence nécessaire du faible développement de la production dans le passé ${ }^{31}$.» Comme la planification étatique de la production devait, selon les marxistes, libérer les forces productives de leurs entraves ${ }^{32}$, la société devait ainsi cesser automatiquement d'être divisée en classes. Ainsi c'est l'État socialiste qui aurait pour fonction de développer rationnellement la production, jusqu'à ce qu'il devienne inutile. Encore une fois le marxisme aboutit dans une conception économiste des rapports sociaux et du développement historique. Par ailleurs cette conception déterministe contredit le volontarisme de la formule consacrée: les hommes font leur propre histoire dans des circonstances déterminées. Elle s'oppose également à la conception de la lutte des classes comme moteur de l'histoire. Seule elle permet d'adhérer à une conception téléologique qui ne s'accorde pas avec la conscience du caractère historiquement déterminé des luttes sociales.

\section{Lénine: un travail de synthèse}

C'est à Lénine que l'on doit d'avoir clarifié et synthétisé les éléments constitutifs de la théorie marxiste de l'État. Lénine tentera aussi d'en lever les ambiguïtés et d'en prolonger les caractères essentiels. En ce sens Lénine enrichit le concept et en clarifie les conséquences, au risque d'en faire apparaître toutes les apories.

On pourrait, à notre avis, résumer sa contribution, contenue dans L'État et la Révolution (1917), par les sept thèses suivantes:

30. Ibid., p. 319.

31. Ibid., p. 317.

32. Dans les faits cette prédiction ne s'est pas réalisée puisque c'est plutôt le capitalisme avancé qui développe les forces productives au rythme le plus rapide. 
1) l'État est le produit de contradictions de classes inconciliables;

2) l'État fonctionne à la violence; il est inséparable de l'existence de détachements spéciaux d'hommes armés;

3) l'État est un instrument d'exploitation de la classe opprimée;

4) l'État bourgeois sera supprimé par le prolétariat au cours d'une révolution violente;

5) tout Etat implique l'existence d'une bureaucratie et d'une armée permanente;

6) la suppression de l'État prolétarien, dont la nécessité provisoire ne peut être mise en doute, se fait sous le mode de l'extinction «graduelle et spontanée»;

7) la période de transition, ou socialisme (première phase du communisme), se caractérise par l'apparition d'une «démocratie nouvelle» et d'une «dictature nouvelle» 33 .

Cette caractérisation de l'État est certes incomplète; elle ne dit rien des fonctions idéologiques de l'État (par exemple pour Gramsci l'Etat fonctionne aussi au consentement), de son action économique ou de son rapport aux formations nationales. Elle est insuffisamment analytique. Par contre elle met en évidence certaines caractéristiques universelles des États : l'État est en effet le réceptacle et l'expression des contradictions sociales, donc des rapports de classes; il comporte invariablement un élément de violence, voire d'extrême violence; il maintient les inégalités sociales les plus fondamentales et sert toujours beaucoup mieux les intérêts des classes dirigeantes qui le dominent, même s'il est souvent le lieu où convergeront les revendications populaires; enfin l'État ne peut se maintenir et remplir ses fonctions sans l'existence d'une bureaucratie importante et d'une armée professionnelle. Seulement ces traits essentiels ne sont pas seulement le propre de l'État bourgeois, ils s'appliquent aussi bien à l'État féodal qu'à l'État socialiste ${ }^{34}$.

33. C'est précisément sur cette question de la coexistence de la démocratie et du socialisme que les critiques les plus virulentes de Rosa Luxembourg contre Lénine et Trotsky portaient.

34. Nous laissons ici de côté la question certes fondamentale de la caractérisation des Etats socialistes soit comme capitalismes d'État ou comme États ouvriers «dégénérés». 
Les problèmes les plus pertinents surviennent avec les deux dernières thèses, encore une fois à propos de la transition et de l'extinction de l'État prolétarien ${ }^{35}$. Mon point de vue est le suivant: l'État est une structure permanente des sociétés développées qui ne peut ni être abolie, ni s'éteindre, ni disparaître; aussi la question de savoir comment l'État pourrait être remplacée comme structure sociale par une autre forme d'organisation relève plus d'une rêveuse utopie que d'un projet concrétisable. Bien sûr cela n'exclut pas que l'État puisse être réformé en profondeur, ou que des États particuliers puissent être détruits et disparaître, mais dans ces cas ce ne sont que des formes particulières de l'État qui sont abolies, non l'État comme structure sociale. Il est tout à fait probable, même dans le cas extrême d'un État moribond, qu'une autre institution étatique prenne promptement la place laissée vacante. Aussi la théorie marxiste sur ce point me semble-t-elle intenable, tant du point de vue de la théorie que du point de vue empirique. Examinons néanmoins la défense léninienne.

Lénine qualifie l'État prolétarien de «demi-État», car « ... il ne faut au prolétariat qu'un État en voie d'extinction, c'est-à-dire constitué de telle sorte qu'il commence immédiatement à s'éteindre et ne puisse pas ne point s'éteindre " ${ }^{35}$. Voilà qu'on aimerait bien voir possible, mais les précisions que Lénine apporte sur la politique de l'État prolétarien sont loin de nous rassurer; en effet, il affirme que cet État devra «mettre en place l'économie socialiste» ${ }^{36},-$ ce qui est déjà un projet de société - , qu'il faudra «instaurer... une discipline de fer maintenue par le pouvoir d'Etat des ouvriers armés » ${ }^{37}$, et enfin que «Marx est centraliste " ${ }^{38}$ au sens de l'unité étatique et nationale. La machine d'État se financera en prélevant un impôt justifié par les mesures sociales qui seront appliquées. Concernant les forces armées, Lénine avance que l'État du prolétariat n'a pas besoin d'armée permanente puisque c'est le peuple en armes qui jouerait ce rôle ; (notons en passant que cette idée a du être promptement abandonnée par les bolchéviks devant la débâcle militaire qui

35. V. LENINE, (4), p. 29.

36. Ibid., p. 61.

37. Ibid.

38. Ibid., p. 66 . 
s'annonçait durant la guerre civile qui succédera à la révolution d'Octobre, Trotsky allant jusqu'à engager certains officiers de l'armée du Tsar pour diriger ses troupes!) Pour ce qui est des autres tâches de l'État, elles devraient se limiter au plan administratif ; cela nécessitera donc une bureaucratie, qui devrait être élue et révocable en tout temps et fonctionner selon le principe des mandats limités dans le temps: on sait que ce mode de fonctionnement sera aussi abandonné très tôt par la République soviétique en raison de l'incurie administrative qui en découle. Tous ces éléments ne militent guère en faveur d'un dépérissement de l'État.

Mais il y a plus important: Lénine va jusqu'à reconnaître qu'en ce qui a trait à la distribution de la richesse sociale l'État bourgeois lui-même subsiste. Il écrit : «Il s'ensuit qu'en régime communiste subsistent pendant un certain temps non seulement le droit bourgeois, mais aussi l'État bourgeois - sans bourgeoisie!» 39 Précisément, - on se rappellera la formule de Engels ${ }^{40}-$, si l'État prolétarien est dans un premier temps un État bourgeois, alors que l'État bourgeois est censé avoir été détruit, si, comme le phénix, sous le régime communiste luimême, l'État bourgeois renaît de ses cendres, on n'a guère de motif d'espérer son dépérissement. Plus radicalement Lénine confirme par là que l'État prolétarien ne saurait être autre chose, dans un premier temps du moins, qu'un Etat capitaliste collectif, ce qui nous rapproche sensiblement des analyses contemporaines qui qualifient les États socialistes de «capitalistes d'État».

Dans un autre passage, cet État apparaît désormais sous une figure qui n'aurait pas trahi l'idéal bureaucratique et totalitaire de Hegel:

Enregistrement et contrôle, tel est l'essentiel, et pour la «mise en route» et pour le fonctionnement régulier de la société communiste dans sa première phase. Ici, tous les citoyens se transforment en employés salariés de l'État constitué par les ouvriers armés. Tous les citoyens deviennent les employés et les ouvriers d'un seul « cartel» du peuple entier, de l'État ${ }^{41}$.

39. Ibid., p. 123.

40. Voir la section 3.2 du présent texte. Engels parle d'un «Etat capitaliste sans capitalistes".

41. Op.c., p. 126. 
Lénine préconise ici rien de moins que l'étatisation de la société dans son ensemble, la centralisation et la concentration des pouvoirs aux mains de l'État et l'identification complète du peuple à l'État; il prétendra ensuite travailler au dépérissement de l'État alors qu'il met en place les prémisses théoriques de l'État le plus centralisateur et le plus hégémonique jamais imaginé. Or cet État, on l'a vu plus haut, serait bourgeois! On croit rêver, mais malheureusement ce n'est pas un rêve : on trouve dans L'Etat et la Révolution une description prophétique du socialisme «effectivement existant», du socialisme d'État tel qu'on a tenté de l'appliquer dans les formations sociales dirigées par les communistes ${ }^{42}$.

Certes Lénine, après Engels, raille la « vénération superstitieuse de l'État» propre aux sociaux-démocrates. Certes il préconise la démocratie pour le peuple. Certes il réclame « tout le pouvoir aux soviets ${ }^{43}$. Mais il refusera aussi de convoquer l'assemblée générale des soviets au moment où les anarchistes risquent d'en ressortir majoritaires, et il n'hésitera pas à les faire massacrer (en 1921) lorsque les marins de Cronstadt se révolteront contre la "dictature marxiste» ${ }^{44}$. On ne peut juger une théorie politique uniquement sur la base de ses bonnes intentions; il faut aussi l'évaluer à la lumière de ses implications pratiques, ce sont les marxistes eux-mêmes qui nous l'ont appris. Ce dernier exemple est particulièrement ironique si on le rapproche de la citation suivante : «... du moment que c'est la majorité du peuple qui mate elle-même ses oppresseurs, il n'est plus besoin d'un "pouvoir spécial» de répression! C'est en ce sens que l'État commence à s'éteindre.» ${ }^{45}$

De fait l'idée d'une extinction de l'État repose sur la base utopique de l'exercice direct du pouvoir par le peuple lui-même. L'histoire a montré que ceci n'est possible que partiellement et pour un temps très court, correspondant à une crise de légitimité et à une perte de contrôle de la part des classes dirigeantes.

42. Une nuance s'impose ici; Lénine avec sa Nouvelle Politique Économique (la N.E.P.), dès le début des années vingt, modèrera radicalement sa politique de socialisation.

43. Comme l'avenir le montrera, ce mot d'ordre avait plus pour but de renverser le gouvernement provisoire que d'accorder une véritable souveraineté populaire aux soviets.

44. Voir l'article Cronstadt, dans le Dictionnaire du mouvement ouvrier, rf. (10), p. 224 à 228.

45. V. LÉNINE, (4), p. 53. 
Lorsqu'un État se constitue, il ne s'éteint pas, il se dresse au contraire «au-dessus» de la société dont il est issu, et tout en la reflétant, il s'autonomise, se renforcit et joue, entre autres choses, un rôle d'oppression, d'exploitation et de gestion des conflits sociaux: qu'un État origine d'une révolution ouvrière ne change rien à ces faits.

\section{Conclusion : le paradoxe marxiste}

Le refus que Marx oppose à ses adversaires de définir les formes futures du socialisme, aura été le trou béant de la théorie marxiste de l'État dans lequel s'engouffrera le «socialisme d'État», par ailleurs présenté pudiquement comme «transitoire» et couvert par le mythe du "dépérissement de l'État». Nous croyons avoir démontré que cette absence et cet aveuglement n'étaient pas fortuits mais nécessaires, parce que liés aux principes premiers de la conception marxiste de la politique d'État. Lorsqu'en marxiste conséquent Lénine, à la suite de Engels, se trouve forcé de définir les caractères et la nature de l'Etat prolétarien, il le définira «malgré lui» comme un État de dictature sociale féroce, centraliste et bureaucratique. De cette définition à ses conséquences pratiques, il n'y a qu'un pas que les marxistes franchiront allègrement ; comme César franchissant le Rubicon, «ils seront venus, ils auront vu et ils auront vaincu».

On connaît les craintes de Lénine sur son lit de mort concernant l'extension incontrôlable du pouvoir de la bureaucratie au détriment des soviets. Mais cette prise de conscience tardive ne suppose pas que la révolution avait été détournée de ses buts, elle se développait au contraire dans la logique même du concept d'État prolétarien tel qu'on le retrouve chez Lénine et chez les fondateurs du marxisme.

La question reste ouverte quant à savoir si une démocratie directe des conseils populaires est possible et viable. Il se peut en effet, comme le pensait Rosa Luxembourg, qu'un État socialiste et démocratique soit réalisable, et que la dégénérescence bureaucratique des États socialistes soit largement liée aux conjonctures de guerre, de misère et au caractère semi-féodal de la plupart des formations sociales ayant passé au socialisme. Mais le modèle marxiste lui-même, comme on l'a vu, prépare et favorise cette 
dégénérescence. On ne peut s'empêcher de rappeler que déjà Bakounine adressait à Marx des mises en garde contre sa théorie de l'État et que ses sombres prédictions devaient se réaliser. Mais pouvait-il en être autrement?

Le paradoxe réside en ceci que la doctrine de l'abolition de la société de classe, le marxisme, est devenu l'idéologie d'une nouvelle classe dominante et la justification d'une oppression étatique sans partage. En ce sens il réalise la projection hégélienne d'une universalité en et pour soi, d'une direction et d'un contrôle absolus de la société civile, de la famille et des individus, désormais soumis à l'État «comme puissance libre», bien mieux qu'il ne s'inspire des réflexions anarchisantes de Marx sur la Commune de Paris. Les États socialistes bureaucratiques ne ramènent-ils pas « la mentalité et toute l'activité de l'individu... à la vie de la substance universelle» ? ${ }^{46} \mathrm{Ne}$ sont-il pas la « réalisation d'une Idée»?

La faiblesse de la théorie marxiste de l'État repose plus profondément sur son incapacité à penser la transformation de l'État autrement que comme un simple changement de forme obéissant à l'évolution de la base économique qui constituerait le véritable contenu. En ce sens le renversement de Hegel n'aurait pas été complété; pour lui l'État n'est-il pas en définitive aussi une forme qui incarne un contenu : celui de l'Esprit rationnel ? La classe prolétarienne serait alors semblable à la conscience de soi, dépouillée et tragique, cheminant vers un accomplissement qui sera en même temps une abolition et un dépassement de soi dans un plus-de-réel. Marx resterait alors prisonnier de la structure métaphysique de la dialectique hégélienne. Le marxisme laisse donc irrésolue la question du socialisme et de l'État, de la démocratie et de l'autonomie du politique.

Département de philosophie, Université du Québec à Montréal.

46. Voir l'exergue introductif. 


\section{OUVRAGES CITÉS}

(1) F. ENGELS, Anti-Dürbing, Ed. Sociales, Paris, 1977.

(2) F. ENGELS, Lettres sur le matérialisme bistorique 1890-1894, Ed. du Progrès, Moscou, 1980.

(3) H. LEFEBVRE, De L'État, T.2, Théorie marxiste de l'État de Hégel à Mao, U.G.E., 10/18, Paris, 1976.

(4) V. LÊNINE, L'État et la Révolution, Ed. en Langues Etrangères, Pékin, 1976.

(5) V. LÉNINE, La Révolution prolétarienne et le renégat Kautsky, Ed. en L.E., Pékin, 1970.

(6) K. MARX et F. ENGELS, Le Manifeste du Parti Communiste, Éd. L.E., Pékin, 1977.

(7) K. MARX, Le Capital, Livre 1, Éd. Soc./Éd. Nouvelles Frontières, Paris/Montréal, 1976.

(8) K. MARX, La Guerre Civile en France 1871, Ed. Soc., Paris, 1968.

(9) K. MARX, Critique du Programme de Gotba, Éd. L.E., Pékin, 1975.

(10) A. NATAF, dir., Dictionnaire du Mouvement Ouvrier, Éd. Universitaires, Paris, 1970. 